\title{
Symmetric Extremal Trajectories in Left-Invariant Optimal Control Problems
}

\section{A. V. Podobryaev}

We consider left-invariant optimal control problems on connected Lie groups. We describe the symmetries of the exponential map that are induced by the symmetries of the vertical part of the Hamiltonian system of the Pontryagin maximum principle. These symmetries play a key role in investigation of optimality of extremal trajectories. For connected Lie groups such that the generic coadjoint orbit has codimension not more than 1 and a connected stabilizer we introduce a general construction for such symmetries of the exponential map.

Keywords: symmetry, geometric control theory, Riemannian geometry, sub-Riemannian geometry

\section{Introduction}

Geometric control theory (see, for example, [1]) deals with left-invariant optimal control problems on a Lie group $G$. Consider a family of left-invariant vector fields $F_{u}$ that depend analytically on $u \in U \subset \mathbb{R}^{n}$. Consider also a left-invariant analytic function $\varphi: G \times U \rightarrow \mathbb{R}$, a point $q_{1} \in G$, and a fixed time $t_{1}>0$. The problem is to find a control $u \in L^{\infty}\left(\left[0, t_{1}\right], U\right)$ and a Lipschitz curve $q_{u}:\left[0, t_{1}\right] \rightarrow G$ such that

$$
\int_{0}^{t_{1}} \varphi\left(q_{u}(t), u(t)\right) d t \rightarrow \min , \quad \dot{q}_{u}(t)=F_{u(t)}\left(q_{u}(t)\right), \quad q_{u}(0)=\mathrm{id}, \quad q_{u}\left(t_{1}\right)=q_{1} \in G .
$$

Consider functions $h_{u}$ on the cotangent bundle $T^{*} G$ that depend on parameter $u \in U$ :

$$
h_{u}(\lambda)=\lambda\left(F_{u}(\pi(\lambda))\right)-\varphi(\pi(\lambda), u), \quad \lambda \in T^{*} G,
$$

Received May 28, 2019

Accepted September 09, 2019

This work was supported by the Russian Science Foundation under grant 17-11-01387 and performed at the A. K. Ailamazyan Program Systems Institute of the Russian Academy of Sciences.

Alexey V. Podobryaev

alex@alex.botik.ru

Ailamazyan Program Systems Institute of RAS

Pereslavl-Zalessky, Yaroslavl Region, 152020 Russia

RUSSIAN JOURNAL OF NONLINEAR DYNAMICS, 2019, 15(4), 569-575 
where $\pi: T^{*} G \rightarrow G$ is the natural projection. Assume that for all $\lambda \in T^{*} G$ the quadratic form $\frac{\partial^{2}}{\partial u^{2}} h_{u}(\lambda)$ is negative definite and the function $u \mapsto h_{u}(\lambda)$ has maximum. Then via the Pontryagin maximum principle $[1,2]$ we obtain a Hamiltonian differential equation on the cotangent bundle $T^{*} G$, such that its phase curves project to optimal trajectories on the group $G$ :

$$
\dot{\lambda}=\vec{H}(\lambda), \quad \pi(\lambda(t))=q_{u}(t), \quad \lambda:\left[0, t_{1}\right] \rightarrow T^{*} G,
$$

where $H(\lambda)=\max _{u \in U} h_{u}(\lambda)$ is the analytic maximized Hamiltonian of the Pontryagin maximum principle, $\vec{H}$ is the corresponding analytic Hamiltonian vector field. The curve $\lambda(t)$ is called a normal extremal. Next we will consider only such extremals. The curve $q_{u}(t)$ is called a normal extremal trajectory.

If we have an explicit solution of the differential equation (1.2), then we have a parametrization of extremal trajectories. After that it remains to study the optimality of extremal trajectories.

Definition 1. A Maxwell point for an optimal control problem (1.1) is a point where two distinct extremal trajectories meet one another with the same value of the cost functional and time. This time is called Maxwell time.

It is well known (see, for example, [12]) that an extremal trajectory cannot be optimal after a Maxwell point. That is why the description of Maxwell points plays an important role in investigating the optimality of extremal trajectories. In particular, the first Maxwell time is an upper bound for the time of loss of optimality (the cut time).

A natural reason of the appearance of Maxwell points is the symmetry of extremal trajectories. Let us give definitions.

Definition 2. The exponential map of problem (1.1) is the map

$$
\operatorname{Exp}: \mathfrak{g}^{*} \times \mathbb{R}_{+} \rightarrow G, \quad \operatorname{Exp}(p, t)=\pi \circ e^{t \vec{H}}(\mathrm{id}, p), \quad(p, t) \in \mathfrak{g}^{*} \times \mathbb{R}_{+},
$$

where $\mathfrak{g}$ is the Lie algebra of the Lie group $G$, and $e^{t \vec{H}}$ is the flow of the Hamiltonian vector field $\vec{H}$.

Definition 3. A symmetry of the exponential map is a pair of diffeomorphisms

$$
s: \mathcal{W} \times \mathbb{R}_{+} \rightarrow \mathcal{W} \times \mathbb{R}_{+}, \quad S: G \rightarrow G \quad \text { such that } \quad \text { Exp } \circ s=S \circ \operatorname{Exp},
$$

where $\mathcal{W} \subset \mathfrak{g}^{*}$ is an open dense subset.

Consider the trivialization of the cotangent bundle via left shifts:

$$
\tau: G \times \mathfrak{g}^{*} \rightarrow T^{*} G, \quad \lambda=\tau(g, p)=d L_{g^{-1}}^{*}(p) \in T_{g}^{*} G, \quad g \in G, \quad p \in \mathfrak{g}^{*}=T_{\mathrm{id}}^{*} G .
$$

where $L_{g}: G \rightarrow G$ is the left shift by the element $g \in G$.

The Hamiltonian $H$ is left-invariant, so we assume that $H \in C^{\infty}\left(\mathfrak{g}^{*}\right)$. A Hamiltonian vector field is a sum of horizontal and vertical parts [1]:

$$
\begin{gathered}
\vec{H}(\tau(g, p))=d_{(g, p)} \tau\left(\vec{H}_{\text {hor }}(g, p)+\vec{H}_{\text {vert }}(p)\right), \\
\vec{H}_{\text {hor }}(g, p)=d L_{g} d_{p} H, \quad \vec{H}_{\text {vert }}(p)=\left(\operatorname{ad}^{*} d_{p} H\right) p,
\end{gathered}
$$

where $d_{p} H \in T_{p}^{*} \mathfrak{g}^{*} \simeq \mathfrak{g}$ is the differential of $H$ at a point $p$. 
The Hamiltonian system $\dot{\lambda}=\vec{H}(\lambda)$ is triangular (its vertical part is independent of state variables). So, one can naturally consider symmetries of the exponential map induced by symmetries of the vertical part of the Hamiltonian system (for a complete statement, see the theorem below).

The plan of investigation of the optimality of extremal trajectories reads as follows.

1. Parametrization of extremal trajectories.

2. Description of symmetries of the vertical part of the Hamiltonian system. Extension of these symmetries to symmetries of the exponential map.

3. Search for Maxwell points that correspond to symmetries. Search for the first Maxwell time as a function $t_{\max }: \mathfrak{g}^{*} \rightarrow \mathbb{R}_{+} \cup\{+\infty\}$.

4. Estimation of the first conjugate time, i.e., the function $t_{\text {conj }}: \mathfrak{g}^{*} \rightarrow \mathbb{R}_{+} \cup\{+\infty\}$ such that a pair $\left(p, t_{\text {conj }}(p)\right)$ is a critical point of the exponential map.

5. Verification of the condition $t_{\max }(p) \leqslant t_{\text {conj }}(p)$ for almost all $p \in \mathfrak{g}^{*}$.

6. Application of the Hadamard theorem on global diffeomorphism [3] to the map

$$
\operatorname{Exp}\left(\cdot, t_{1}\right):\left\{p \in \mathfrak{g}^{*} \backslash 0 \mid t_{1}<t_{\max }(p)\right\} \rightarrow G \backslash(\{\operatorname{id}\} \cup \overline{\mathcal{M}}),
$$

where $\overline{\mathcal{M}}$ is the closure of the Maxwell set.(A smooth nondegenerate proper map of connected and simply connected manifolds of equal dimensions is a diffeomorphism.)

We need items 4 and 5 to verify the nondegeneracy condition of the Hadamard theorem. If the implementation of all these steps is complete, than the first Maxwell time is actually the cut time.

Notice that the implementation of this program is not guaranteed. For example, the symmetries of the vertical part of the Hamiltonian system may not produce a complete description of the Maxwell set. Such a situation appears in the Euler elasticae problem [4, 5]. However, this method works in several sub-Riemannian [6] and Riemannian problems (see references below):

1. Sub-Riemannian problem on Heisenberg type group (C. Autenried, M. Godoy Molina [7]).

2. Free nilpotent sub-Riemannian problem with growth vector $(3,6)$ (O. Myasnichenko [8] and independently A. Montanari and D. Morbidelli [9], also some results in the general case of free step two Carnot group achieved by L. Rizzi and U. Serres $[10])$.

3. Generalized Dido problem (Yu. L. Sachkov [11-13]).

4. Sub-Riemannian problem on Engel group (A. A. Ardentov, Yu. L. Sachkov [14-17]).

5. Sub-Riemanian problems on the Lie groups $\mathrm{SL}_{2}(\mathbb{R}), \mathrm{PSL}_{2}(\mathbb{R}), \mathrm{SO}_{3}, \mathrm{SU}_{2}$

(U. Boscain, F. Rossi [18], and independently, using another techniques by V. N. Berestovskii and I. A. Zubareva [19, 20]), also C. Autenried and I. Markina considered some generalizations to Stiefel manifolds [21].

6. Riemannian problems on the Lie groups $\mathrm{SL}_{2}(\mathbb{R}), \mathrm{PSL}_{2}(\mathbb{R}), \mathrm{SO}_{3}, \mathrm{SU}_{2}$ (A. V. Podobryaev, Yu. L. Sachkov $[22,23])$.

7. Sub-Riemannian problem on the Lie group $\mathrm{SE}_{2}$ (Yu. L. Sachkov [24-26], the first paper in collaboration with I. Moiseev).

8. Sub-Riemannian problem on the Lie group $\mathrm{SH}_{2}$ (Ya. A. Butt, Yu. L. Sachkov, A. I. Bhatti $[27,28])$.

9. The problem of a rolling sphere on the plane without twisting and slipping (Yu. L. Sachkov [29]). 
In the problems listed above an extension of symmetries of the vertical subsystem to symmetries of the exponential map was constructed by explicit formulas for the map Exp (i.e., an explicit parametrization of extremal trajectories) or by an explicit form of the Hamiltonian system. The existence of such an extension was not guaranteed a priori.

In Section 2 we introduce conditions for existence of the extension of symmetries of the vertical subsystem to symmetries of the exponential map. Also, there is a general construction of such symmetries and some corollaries. In Section 3 we give a brief description of the proof.

\section{The main result}

Theorem. Let $G$ be a connected Lie group, such that the generic stabilizer of the coadjoint action is connected and has dimension not more than 1. Assume that $H: T^{*} G \rightarrow \mathbb{R}$ is a leftinvariant Hamiltonian, and an operator $\sigma^{*}: \mathfrak{g}^{*} \rightarrow \mathfrak{g}^{*}$ is such that $\sigma^{*}$ preserves the Hamiltonian $H$ and one of the following two conditions holds:

(a) $\sigma^{*}\left(\vec{H}_{\text {vert }}\right)=\vec{H}_{\text {vert }}$ and $\sigma$ is an automorphism of the Lie algebra $\mathfrak{g}$;

(b) $\sigma^{*}\left(\vec{H}_{\text {vert }}\right)=-\vec{H}_{\text {vert }}$ and $\sigma$ is an antiautomorphism of the Lie algebra $\mathfrak{g}$.

Then the pair of diffeomorphisms $\left(s, S^{-1}\right)$ is a symmetry of the exponential map, where

$$
s(p, t)= \begin{cases}\left(\sigma^{*} p, t\right), & \text { in case }(\mathrm{a}), \\ \left(\sigma^{*} e^{t \vec{H}_{\mathrm{vert}}} p, t\right), & \text { in case }(\mathrm{b}),\end{cases}
$$

and $S: G \rightarrow G$ is the (anti)automorphism of the Lie group such that $d_{\mathrm{id}} S=\sigma$.

REMARK 1. The condition for the generic stabilizer of the coadjoint action plays a role only for symmetries of case (b).

REMARK 2. In case (b), if $\sigma$ is an antiautomorphism, then $-\sigma$ is an automorphism and one can construct a symmetry as in case (a). But it is not what we want. We construct not any symmetry, but a special kind of symmetry for case (b). Notice that in case (b) the curve

$$
\left\{\operatorname{Exp} \circ s(p, \tau)=\pi \circ e^{\tau \vec{H}} \circ \sigma^{*} \circ e^{\tau \vec{H}_{\text {vert }}}(\mathrm{id}, p) \mid \tau \in \mathbb{R}_{+}\right\}
$$

is not an extremal trajectory, unlike the situation in case (a). The segment of extremal trajectory $\left\{\operatorname{Exp}\left(\sigma^{*} e^{t \vec{H}_{\text {vert }}} p, \tau\right) \mid \tau \in[0, t]\right\}$ is symmetric to the segment of the initial extremal trajectory $\{\operatorname{Exp}(p, \tau) \mid$ $\tau \in[0, t]\}$.

Usually the symmetries of case (b) are essential for construction of Maxwell strata in applications. In examined situations there is an open subset of extremal trajectories that intersect the corresponding Maxwell stratum.

Everywhere below we consider symmetries $\left(s, S^{-1}\right)$ of the exponential map such that $\sigma$ satisfies the hypotheses of Theorem 2. We call $\sigma^{*}$ the symmetry of the vertical part of the Hamiltonian vector field.

Definition 4. The Maxwell sets in the preimage and in the image of exponential map corresponding to the symmetry $\left(s, S^{-1}\right)$ are the sets

$$
M_{\sigma^{*}}=\left\{\left(p, t_{1}\right) \in \mathcal{W} \times \mathbb{R}_{+} \mid \operatorname{Exp}\left(p, t_{1}\right)=\operatorname{Exp} \circ\left(p, t_{1}\right)\right\}, \quad \mathcal{M}_{\sigma^{*}}=\operatorname{Exp} M_{\sigma^{*}},
$$

respectively. 
REMARK 3. It follows directly from Theorem 2 that the Maxwell set in the image of the exponential map $\mathcal{M}_{\sigma^{*}}$ is a subset of the set of fixed points $G^{S}$ of the mapping $S$. Let $F_{\sigma^{*}}(g)=0$ be an equation of $G^{S}$, where $F_{\sigma^{*}}: G \rightarrow \mathbb{R}^{k}$, for some $k$. This allows us to determine the Maxwell time $t_{\max }^{\sigma^{*}}: \mathfrak{g}^{*} \rightarrow \mathbb{R}_{+} \cup\{+\infty\}$, corresponding to the symmetry $\sigma^{*}$, by the implicit function

$$
F_{\sigma^{*}}\left(\operatorname{Exp}\left(p, t_{\max }^{\sigma^{*}}(p)\right)\right)=0 .
$$

To find the set of the first Maxwell points corresponding to symmetries we need to investigate these implicit functions for different symmetries to find the first Maxwell time.

Corollary 1. Assume that there are two left-invariant optimal control problems on a Lie group and a symmetry of the vertical parts of both Hamiltonian vector fields. If two extremal trajectories corresponding to these problems meet one another, then the symmetric trajectories meet one another as well with the same values of time and cost functional.

Corollary 2. Let $G$ be a connected compact Lie group. If a normal extremal trajectory meets a geodesic of the Killing metric, then the symmetric extremal trajectory meets the symmetric geodesic of the Killing metric at the same instant of time.

Proof. Corollary 1 immediately follows from Theorem 2.

The vertical part of the Hamiltonian vector field is trivial for the Riemannian problem for the Killing metric (see, for example, [1]). Thus, any symmetry of the vertical part of the Hamiltonian system for the optimal control problem is a symmetry of the vertical part of the Hamiltonian vector field for the Riemannian problem for the Killing metric. Corollary 2 follows from Corollary 1.

REMARK 4. For any element $g \in G$ of a compact connected Lie group there exists $\xi \in \mathfrak{g}$ such that $g=\exp (\xi)$. By definition we have $S^{-1}(g)=\exp \left(\sigma^{-1} \xi\right)$. This does not depend on a choice of the element $\xi$, because the (anti)automorphism $\sigma$ preserves fibers of the map exp. For a compact Lie group $G$ one-parametric subgroups $g_{\xi}(t)=\exp (t \xi)$ are geodesics of the bi-invariant Riemannian metric that is defined by the Killing form [1].

\section{Brief description of the proof of the theorem}

Define the diffeomorphism $S^{*}: T^{*} G \rightarrow T^{*} G$ as follows:

$$
S^{*}(\lambda)=\left(d_{S^{-1}(g)} S\right)^{*}(\lambda), \quad \lambda \in T^{*} G, \quad \pi(\lambda)=g \in G .
$$

By definition we have

$$
\pi \circ S^{*}=S^{-1} \circ \pi,\left.\quad S^{*}\right|_{T_{\mathrm{id}}^{*} G}=\sigma^{*} .
$$

Moreover, $S^{*}$ is a symplectomorphism of the canonical symplectic structure $\omega$ on $T^{*} G$ and $\left(S^{*}\right)^{-1} \circ e^{t \vec{H}} \circ S^{*}=e^{\overline{t\left(H \circ S^{*}\right)}}$.

Next we find out how the diffeomorphism $S^{*}$ acts on the Hamiltonian $H$.

It is easy to see that if $\sigma$ is an automorphism of the Lie algebra $\mathfrak{g}$ and $\sigma^{*}$ preserves the left-invariant Hamiltonian $H$, then $H \circ S^{*}=H$.

As a consequence in case (a) we obtain $e^{t \vec{H}} \circ S^{*}=S^{*} \circ e^{t \vec{H}}$. From equalities (3.1) it follows that $\operatorname{Exp} \circ s=S^{-1} \circ \operatorname{Exp}$. So, the pair $\left(s, S^{-1}\right)$ is a symmetry of exponential map in case (a).

Consider now case (b). If $\sigma$ is an antiautomorphism of the Lie algebra $\mathfrak{g}$ and $\sigma^{*}$ preserves the left-invariant Hamiltonian $H$, then the function $H_{R}=H \circ S^{*}$ is right-invariant. The corresponding Hamiltonian vector field $\vec{H}_{R}$ is right-invariant and its vertical part in the right trivialization of the cotangent bundle is equal to $-\vec{H}_{\text {vert }}$. 
Next we use the following notation. For $p \in \mathfrak{g}^{*}$ denote by $G_{p}=\left\{g \in G \mid\left(\operatorname{Ad}^{*} g\right) p=p\right\}$ the stabilizer of the covector $p$ with respect to the coadjoint action. Let $\mathfrak{g}_{p}$ be the Lie algebra of the stabilizer $G_{p}$.

Lemma. Assume that $p_{0} \in \mathfrak{g}^{*}$ is such that the stabilizer $G_{p_{0}}$ is connected, $\operatorname{dim} G_{p_{0}} \leqslant 1$ and $p_{0}\left(\mathfrak{g}_{p_{0}}\right) \neq 0$. Then

$$
e^{t \vec{H}_{R}} \circ e^{t \vec{H}_{\text {vert }}} p_{0}=e^{t \vec{H}} p_{0} \quad \text { for all } \quad t \in \mathbb{R}_{+} .
$$

The idea of the proof is to consider the momentum maps of left- and right- actions of the group $G$ on the cotangent bundle $T^{*} G$. These momentum maps are principal $G_{p_{0}}$-bundles over the coadjoint orbit $\left(\mathrm{Ad}^{*} G\right) p_{0}$. The fibers of these bundles are one-dimensional (due to the assumption of the lemma).

This allows us to introduce principal connections on these bundles. Those principal connections are produced by canonical Liouville one-form on the cotangent bundle.

Next, we compute the horizontal and vertical components (with respect to the principal connections) of the flows of Hamiltonian vector fields $\vec{H}$ and $\vec{H}_{R}$ and obtain the statement of the lemma.

Finally, notice that $\left\{p \in \mathfrak{g}^{*} \mid p\left(\mathfrak{g}_{p}\right) \neq 0\right\}$ is an open dense subset in $\mathfrak{g}^{*}$. Consider the open dense subset $\mathcal{W}=\left\{p \in \mathfrak{g}^{*} \mid \operatorname{codim}\left(\operatorname{Ad}^{*} G\right) p=1, p\left(\mathfrak{g}_{p}\right) \neq 0\right\} \subset \mathfrak{g}^{*}$. Define symmetry $s$ on this subset in the preimage of exponential map. We have $e^{t \vec{H}}(p)=e^{t \vec{H}_{R}} \circ e^{t \vec{H}_{\text {vert }}}(p)$ for $p \in \mathcal{W}$. It follows that this is equal to $\left(S^{*}\right)^{-1} \circ e^{t \vec{H}} \circ S^{*} \circ e^{t \vec{H}_{\mathrm{vert}}}$, and we obtain $e^{t \vec{H}} \circ S^{*} \circ e^{t \vec{H}_{\mathrm{vert}}}(p)=$ $=S^{*} \circ e^{t \vec{H}}(p)$ for $p \in \mathcal{W}$. Now the theorem follows from (3.1).

\section{References}

[1] Agrachev, A. A. and Sachkov, Yu. L., Control Theory from the Geometric Viewpoint, Encyclopaedia Math. Sci., vol. 87, Berlin: Springer, 2004.

[2] Pontryagin, L. S., Boltyanskii, V. G., Gamkrelidze, R. V., and Mishchenko, E. F., The Mathematical Theory of Optimal Processes, L. W. Neustadt (Ed.), New York: Wiley, 1962.

[3] Krantz, S. G. and Parks, H. R., The Implicit Function Theorem: History, Theory, and Applications, Boston, Mass.: Birkhäuser, 2002.

[4] Sachkov, Yu. L., Maxwell Strata in Euler's Elastic Problem, J. Dyn. Control Syst., 2008, vol. 14, no. 2, pp. 169-234.

[5] Ardentov, A. A., Multiple Solutions in Euler's Elastic Problem, Autom. Remote Control, 2018, vol. 79, no. 7, pp. 1191-1206; see also: Avtomat. i Telemekh., 2018, no. 7, pp. 22-40.

[6] Agrachev, A., Barilari, D., and Boscain, U., A Comprehensive Introduction to Sub-Riemannian Geometry, Cambridge Stud. Adv. Math., vol. 181, Cambridge: Cambridge Univ. Press, 2019.

[7] Autenried, C. and Godoy Molina, M., The Sub-Riemannian Cut Locus of $H$-Type Groups, Math. Nachr., 2016, vol. 289, no. 1, pp. 4-12.

[8] Myasnichenko, O., Nilpotent (3,6) Sub-Riemannian Problem, J. Dyn. Contr. Syst., 2002, vol.8, no. 4, pp. 573-597.

[9] Montanari, A. and Morbidelli, D., On the subRiemannian cut locus in a model of free two-step Carnot group, Calc. Var. Partial Dif., 2017, vol. 56, no. 2, Art. 36.

[10] Rizzi, L. and Serres, U., On the Cut Locus of Free, Step Two Carnot Groups, Proc. Amer. Math. Soc., 2017, vol. 145, no. 12, pp. 5341-5357.

[11] Sachkov, Yu. L., Discrete Symmetries in the Generalized Dido Problem, Sb. Math., 2006, vol. 197, nos. 1-2, pp. 235-257; see also: Mat. Sb., 2006, vol.197, no. 2, pp. 95-116. 
[12] Sachkov, Yu. L., The Maxwell Set in the Generalized Dido Problem, Sb. Math., 2006, vol.197, nos. 3-4, pp. 595-621; see also: Mat. Sb., 2006, vol.197, no.4, pp. 123-150.

[13] Sachkov, Yu. L., Complete Description of the Maxwell Strata in the Generalized Dido Problem, $S b$. Math., 2006, vol. 197, nos.5-6, pp.901-950; see also: Mat. Sb., 2006, vol.197, no. 6, pp. 111-160.

[14] Ardentov, A. A. and Sachkov, Yu. L., Extremal Trajectories in the Nilpotent Sub-Riemannian Problem on the Engel Group, Sb. Math., 2011, vol.202, nos.11-12, pp. 1593-1615; see also: Mat. Sb., 2011, vol. 202, nos. 11, pp. 31-54.

[15] Ardentov, A.A. and Sachkov, Yu. L., Conjugate Points in Nilpotent Sub-Riemannian Problem on the Engel Group, J. Math. Sci. (N. Y.), 2013, vol. 195, no. 3, pp. 369-390.

[16] Ardentov, A. A. and Sachkov, Yu. L., Cut Time in Sub-Riemannian Problem on Engel Group, ESAIM Control Optim. Calc. Var., 2015, vol. 21, no.4, pp. 958-988.

[17] Ardentov, A. A. and Sachkov, Yu. L., Maxwell Strata and Cut Locus in Sub-Riemannian Problem on Engel group, Regul. Chaotic Dyn., 2017, vol. 22, no. 8, pp. 909-936.

[18] Boscain, U. and Rossi, F., Invariant Carnot-Carathéodory Metrics on $S^{3}, \mathrm{SO}(3)$, SL(2) and Lens Spaces, SIAM J. Control Optim., 2008, vol.47, no. 4, pp. 1851-1878.

[19] Berestovskii, V. N. and Zubareva, I. A., Geodesics and Shortest Arcs of a Special Sub-Riemannian Metric on the Lie Group SL(2), Siberian Math. J., 2016, vol.57, no. 3, pp.411-424; see also: Sibirsk. Mat. Zh., 2016, vol.57, no. 3, pp. 527-542.

[20] Berestovskii, V. N. and Zubareva, I. A., Geodesics and Shortest Arcs of a Special Sub-Riemannian Metric on the Lie Group SO(3), Siberian Math. J., 2015, vol.56, no. 4, pp.601-611; see also: Sibirsk. Mat. Zh., 2015, vol.56, no. 4, pp. 762-774.

[21] Autenried, C. and Markina, I., Sub-Riemannian Geometry of Stiefel Manifolds, SIAM J. Control Optim., 2014, vol. 52, no. 2, pp. 939-959.

[22] Podobryaev, A. V. and Sachkov, Yu. L., Cut Locus of a Left Invariant Riemannian Metric on SO(3) in the Axisymmetric Case, J. Geom. Phys., 2016, vol. 110, pp. 436-453.

[23] Podobryaev, A. V. and Sachkov, Yu. L., Symmetric Riemannian Problem on the Group of Proper Isometries of Hyperbolic Plane, J. Dyn. Control Syst., 2018, vol. 24, no. 3, pp. 391-423.

[24] Moiseev, I. and Sachkov, Yu. L., Maxwell Strata in Sub-Riemannian Problem on the Group of Motions of a Plane, ESAIM Control Optim. Calc. Var., 2010, vol. 16, pp. 380-399.

[25] Sachkov, Yu. L., Conjugate and Cut Time in the Sub-Riemannian Problem on the Group of Motions of a Plane, ESAIM Control Optim. Calc. Var., 2010, vol. 16, pp. 1018-1039.

[26] Sachkov, Yu. L., Cut Locus and Optimal Synthesis in the Sub-Riemannian Problem on the Group of Motions of a Plane, ESAIM Control Optim. Calc. Var., 2011, vol.17, pp. 293-321.

[27] Butt, Ya. A., Sachkov, Yu. L., and Bhatti, A. I., Maxwell Strata and Conjugate Points in the SubRiemannian Problem on the Lie Group SH(2), J. Dyn. Control Syst., 2016, vol. 22, no. 4, pp. 747-770.

[28] Butt, Ya. A., Sachkov, Yu. L., and Bhatti, A. I., Cut Locus and Optimal Synthesis in Sub-Riemannian Problem on the Lie Group SH(2), J. Dyn. Control Syst., 2017, vol. 23, no. 1, pp. 155-196.

[29] Sachkov, Yu. L., Maxwell Strata and Symmetries in the Problem of Optimal Rolling of a Sphere over a Plane, Sb. Math., 2010, vol. 201, no.7, pp. 1029-1051; see also: Mat. Sb., 2010, vol. 201, no. 7, pp. 99-120. 